\title{
The effect of $\mathrm{Nb}$ on the high strain rate hydrogen embrittlement of Q\&P steel
}

\author{
Florian Vercruysse ${ }^{1 *}$, Lisa Claeys $^{2}$, Tom Depover $^{2}$, Kim $_{\text {Verbeken }}^{2}$, Patricia Verleysen ${ }^{1}$, \\ and Roumen Petrov ${ }^{1,3}$ \\ ${ }^{1}$ Department of Electromechanical, Systems and Metal Engineering, Ghent University, \\ Technologiepark 46, 9052 Zwijnaarde, Belgium \\ ${ }^{2}$ Department of Materials, Textiles and Chemical Engineering, Ghent University, \\ Technologiepark 46, 9052 Zwijnaarde, Belgium \\ ${ }^{3}$ Department of Materials Science and Engineering, Delft University of Technology, \\ Mekelweg 2, 2628CD Delft, The Netherlands
}

\begin{abstract}
Quenching and Partitioning (Q\&P) steels are, due to their excellent combination of strength and ductility, seen as good candidates for the third generation advanced high strength steels (AHSS). Although the TRIP effect is beneficial for the overall mechanical behaviour of these steels it potentially can have detrimental effects when strained in a hydrogenenriched environment. The solubility of hydrogen is high in austenite but low in high carbon martensite. Martensite is even in the absence of hydrogen already a possible damage initiation spot. The effect of hydrogen under static and dynamic tensile loading was evaluated in a Q\&P and a $\mathrm{Nb}$ micro-alloyed Q\&P steel. Experiments were carried out under a strain rate ranging from $0.03 \mathrm{~s}^{-1}$ till $500 \mathrm{~s}^{-1}$ and correlated with the hydrogen uptake characterised via thermal desorption spectroscopy (TDS). The presence of $\mathrm{Nb}$ resulted in a $25 \%$ increase in the hydrogen uptake capacity. A higher susceptibility to hydrogen was observed in the $\mathrm{Nb}$ steel partially due to the high hydrogen fraction, but also because of the larger fraction of low stability austenite. However, when tested under dynamic conditions the hydrogen susceptibility is minor and even improved in the micro-alloyed Q\&P steel compared to the standard Q\&P steel.
\end{abstract}

\section{Introduction}

Research into new and better materials is continuously ongoing due to the constant challenge of keeping up with the increasingly stringent emissions regulations and the high passenger safety standard in the automotive industry. To this purpose, the first generation of advanced high strength steels (AHSS) was introduced in the 1970's. Later research resulted in the second generation, characterized by a significant increase in both strength and ductility, but

\footnotetext{
* Corresponding author: Florian.Vercruysse@UGent.be
} 
with the main drawback that the application domain of these $2^{\text {nd }}$ generation steels was rather limited due to their dependence on high fractions of expensive alloying elements. In recent years, several steels show promising properties to claim to be part of the $3^{\text {rd }}$ generation AHSS. This generation has properties situated between the first and the second, but with a quite lean alloying strategy. Quenching and Partitioning (Q\&P) steels are one of these $3^{\text {rd }}$ generation AHSS.

Q\&P steels, first introduced by Speer, obtain their excellent combination of strength and ductility, and thus energy absorption potential, due to a microstructure containing two phases. Tempered martensite is the main phase and is supplemented with a significant fraction (10$15 \%)$ of retained austenite [1]. The high strength provided by the martensite matrix coupled with the high ductility as a result of the TRIP effect due to the presence of retained austenite is the reason behind the excellent energy absorption potential of these steels. Further, it is known that the mechanical behaviour of steels can change significantly when the strain rate is increased. To date, the number of studies on the dynamic behaviour of Q\&P steels is fairly limited

The microstructure is created in a two-step thermal cycle. After complete or partial austenitization, the material is quenched to a temperature below the martensite start temperature Ms, but above the martensite finish temperature Mf to transform a certain fraction of austenite into martensite. The fraction formed is completely determined by the quenching temperature. Afterwards, the material is heated up to a partitioning temperature to allow the excess carbon to diffuse from the supersaturated martensite to austenite as a result of the addition of $\mathrm{Si}$. The latter is reported to kinetically suppress the formation of cementite allowing for the carbon to diffuse to austenite. The increase of carbon in austenite has a stabilizing effect. Upon sufficient carbon enrichment, the Ms temperature drops below room temperature. As a result, the austenite is retained when cooling to room temperature. Insufficient enrichment will result in the formation of fresh martensite during the final quench, causing potential early damage initiation [2].

Numerous times, small grains have been reported to be beneficial for a wide range of the steel applications, because they ensure both high strength and high toughness. Several studies reported on the addition of micro-alloying elements as a possible strategy for grain refinement. However, research into the addition of micro-alloying elements like $\mathrm{Ti}, \mathrm{Nb}$ or $\mathrm{V}$ in Q\&P steels is still limited [3].

High strength steels are reported to frequently suffer from premature failure in hydrogenrich environments. This is seen as a crucial issue for the application of AHSS in several industrial fields. Next to the fact that AHSS are often highly susceptible to hydrogen embrittlement (HE), Q\&P steels potentially might be characterized by a higher susceptibility due to the presence of retained austenite. The hydrogen solubility in austenite is significantly higher than in martensite. The TRIP effect, which is the reason behind the excellent ductility of these steels, results in a high hydrogen fraction in the vicinity of freshly formed martensite. Fresh martensite that is already prone to early damage initiation can potentially have a detrimental effect on the mechanical properties of Q\&P steels when operated in hydrogenrich environments. The stability of austenite might play an important role in the susceptibility to hydrogen-induced failure.

Although some research has been carried out on the effect of the stability of austenite on hydrogen susceptibility, it is still not completely understood. In earlier publications of the authors, it was reported that imposing a high strain rate, as would be the case in a car crash, affects the TRIP effect due to the adiabatic heating influencing the stability of austenite [4]. Therefore, the present research is focused on the effect of the addition of $\mathrm{Nb}$ on the $\mathrm{HE}$ susceptibility of a Q\&P steel under static and dynamic loading conditions. To evaluate the effect of $\mathrm{Nb}$, steels with and without $\mathrm{Nb}$ were investigated. 


\section{Materials and experimental techniques}

\subsection{Materials}

Two steels, a $\mathrm{Nb}$ free and $\mathrm{Nb}$ steel, were studied. The chemical compositions of both steels are presented in Table 1.

Table 1: Chemical composition of steel used (wt \%)

\begin{tabular}{llllllll}
\hline & C & Si & Mn & Al & Cr & Nb & Fe \\
\hline $\begin{array}{l}\text { Nb free } \\
\text { steel }\end{array}$ & 0.199 & 1.260 & 2.350 & 0.018 & 0.025 & - & Bal. \\
Nb steel & 0.192 & 1.260 & 2.410 & 0.019 & 0.300 & 0.024 & Bal. \\
\hline
\end{tabular}

After casting, the steels, with the composition as described above, were hot rolled and subsequently cold rolled to a final thickness of $1.5 \mathrm{~mm}$. The total cold reduction applied to the steels is $50 \%$. The Q\&P heat treatment cycle is based on dilatometric experiments to minimize the fresh martensite fraction. The samples were autenitized at $870^{\circ} \mathrm{C}$ for $100 \mathrm{~s}$ to homogeneously distribute the alloying elements prior to quenching to $290^{\circ} \mathrm{C}$ or $300^{\circ} \mathrm{C}$ for resp. the $\mathrm{Nb}$ and the $\mathrm{Nb}$ free steel. The discrepancy between the two quenching temperatures is the result of the lowering effect of $\mathrm{Cr}$ and $\mathrm{Nb}$ on the Ms temperature [5], [6]. Both steels were partitioned at $400^{\circ} \mathrm{C}$ for $50 \mathrm{~s}$. The thermal homogeneity of the treatment was monitored by several K1 thermocouples welded to the plate. A final homogenously heat treated zone of $100 \times 200 \mathrm{~mm}^{2}$ was achieved from which all the samples were extracted for microstructural and mechanical analysis.

\subsection{Experimental techniques}

\subsubsection{Hydrogen charging and characterisation}

Thermal desorption spectroscopy (TDS), after electrochemically charging in $0.5 \mathrm{M}$ sulphuric acid for four hours at $0.8 \mathrm{~mA} / \mathrm{cm}^{2}$, was used to study the hydrogen desorption kinetics in the materials as well as to evaluate the hydrogen concentration within each steel. The hydrogen desorption kinetics can be used to determine the hydrogen traps present in the material as well as to provide information on the fraction of the traps. Hydrogen will diffuse out of the samples when the temperature is sufficiently high to overcome the desorption activation energy of the traps. The hydrogen atoms forming $\mathrm{H}_{2}$-gas leaving the sample are extracted via a carrier gas $\left(\mathrm{N}_{2}\right)$ forced over the sample and analysed by a mass spectrometer. This results in a flux of hydrogen leaving the sample as a function of the temperature.

\subsubsection{Mechanical characterisation}

To characterise the quasi-static mechanical properties, a conventional Instron ${ }^{\mathrm{TM}} 5569$ tensile test device equipped with a $50 \mathrm{kN}$ load cell was used. The tensile tests were performed at a nominal strain rate of $0.3 \mathrm{~s}^{-1}$.

Split Hopkinson Tensile Bar (SHTB) experiments were performed to characterise the mechanical properties under dynamic deformation [7]. During the SHTB tests, the samples were fixed between two long bars and loaded by a mechanical tensile wave. The amplitude of the tensile wave can be adjusted, which allows controlling the strain rate in the sample. For all dynamic experiments, a strain rate of approximately $500 \mathrm{~s}^{-1}$ was aimed at. SHTB tests require the use of samples with reduced dimensions [8]. Therefore, sub-sized tensile dogbone-shaped samples with a gauge length of $6 \mathrm{~mm}$ and gauge width of $3 \mathrm{~mm}$ were used. 
The samples were fixed to the test bench with pins. The samples were extracted along the rolling direction of the cold rolled sheet.

Dynamic testing is characterised by a short duration of the test. The increase in temperature due to adiabatic heating is estimated assuming that the heat generated by plastic work in the sample is not dissipated to the environment. For steels, generally, a value of 0.9 is adopted for the fraction of the plastic work converted into heat, denominated as the TaylorQuinney coefficient. However, as the transformation of austenite to martensite is exothermic, in present study, a coefficient equal to 1 was used instead of 0.9 to, at least approximately, account for the heat production due to the transformation [9].

All tensile tests were carried out ex-situ the H-charging chamber. The tensile tests, irrespective of the strain rate applied, were conducted at a fixed time of 4 minutes after the samples were taken from the charging set up. The embrittlement index (EI) used to evaluate the effect of hydrogen on the ductility of the material is derived as follows:

$$
E I=\frac{\epsilon_{f}^{\text {Air }}-\epsilon_{f}^{H}}{\epsilon_{f}^{\text {Air }}}
$$

where $\epsilon_{f}^{\text {Air }}$ and $\epsilon_{f}^{H}$ are resp. the engineering strain at fracture for the reference samples tested in air and the H-charged samples.

\section{Results and discussion}

\subsection{Hydrogen charging and characterization}

TDS analysis after charging indicates an increase in the hydrogen uptake capacity by the addition of $\mathrm{Nb}$. Resp. 5 and 4 ppm $\mathrm{H}$ is absorbed in the $\mathrm{Nb}$ and the $\mathrm{Nb}$ free sample. This $25 \%$ increase in hydrogen uptake is explained by taken the refining effect of $\mathrm{Nb}$ on the microstructure into account. The addition of $0.025 \mathrm{wt} \% \mathrm{Nb}$ resulted in the average martensite grain diameter being reduced in size from $3.7 \mu \mathrm{m}$ to $2.7 \mu \mathrm{m}$. On top of this, the retained austenite is characterized by a smaller grain size as well, as is reported in prior work [10]. The more refined structure results in a higher fraction of grain and phase boundaries. These boundaries can, as they are a defect in the material, be seen, next to e.g. dislocations, as trapping sites for hydrogen. The higher fraction of these trapping sites, therefore, explains the higher hydrogen uptake seen in the $\mathrm{Nb} \mathrm{Q} \& \mathrm{P}$ steel.

\subsection{Static and dynamic tensile behaviour}

The embrittlement index of the static and dynamic experiments can be seen in Figure 1. The presence of resp. 4 and 5ppm $\mathrm{H}$ for the $\mathrm{Nb}$ free and $\mathrm{Nb}$ steel has clearly caused a significant reduction in the ductility of the steel when tested under static conditions (strain rate 0.003 $\left.0.03 \mathrm{~s}^{-1}\right)$. The addition of $\mathrm{Nb}$ to the Q\&P steel, next to the general increase in the strength (UTS) and strain hardening rate of the steel, caused the steel to have a higher susceptibility to hydrogen embrittlement [10]. The Nb steel suffers more (EI of 57\% compared to 44\%) in comparison to the $\mathrm{Nb}$ free steel. The higher EI is partially attributed to the higher fraction of hydrogen in the sample, as observed earlier, but also to the higher austenite fraction. The $\mathrm{Nb}$ addition resulted in an increase of the retained austenite fraction from $10 \%$ to $13 \%$, but the austenite is generally of lower stability [10]. The higher fraction of low stability austenite results in the higher fraction of high carbon martensite being formed in the $\mathrm{Nb}$ steel when compared to the $\mathrm{Nb}$ free steel upon straining. This high carbon martensite, which is known to be brittle, and therefore more susceptible to Hydrogen-induced failure, in combination 
with the higher fraction of hydrogen present are the main reasons for the increased EI in the $\mathrm{Nb}$ Q\&P steel.

Hydrogen in the material reduces the ductility of the material by diffusing to the stress zones in front of defects e.g. crack tips and affects the cohesion of the atoms and shields the stress fields of dislocations from each other. All mechanisms result in premature and brittle fracture. However, before this can occur, hydrogen has to diffuse to the crack tips or damage initiation spots. This dependency on diffusion results in the fact that the extent of the hydrogen-induced embrittlement is affected by the strain rate. Higher strain rates often reduce the susceptibility of the material to hydrogen-induced failure. Both Q\&P steels were tested here under dynamic conditions and the results are presented in Figure 1 as well. The EI is not only reduced, though the embrittlement is also almost negligible. Only a reduction in ductility of a few percent is observed. This indicates the importance of diffusion and therefore strain rate imposed on the material. However, in both steels, next to the reduced diffusion time, a secondary effect is resulting in the excellent high strain rate behaviour when tested in a hydrogen-rich environment. The adiabatic heating, as a result of the high strain rate conditions, raises the sample temperature by more than $80^{\circ} \mathrm{C}$. The increase in temperature has a stabilizing effect on retained austenite. The lower fraction of austenite transforming to harmful, high carbon fresh martensite further explains the reduction in EI by increasing the deformation speed into the dynamic range. Although both steels are characterized by a limited reduction in ductility, the addition of $\mathrm{Nb}$ seems to have further lowered the hydrogen susceptibility when tested under dynamic conditions. This is attributed to the proportionally higher temperature increase in the $\mathrm{Nb}$ steel when compared to the $\mathrm{Nb}$ free steel resulting from the higher strength. The higher temperature increase further stabilises the RA and therefore explains the lower EI.

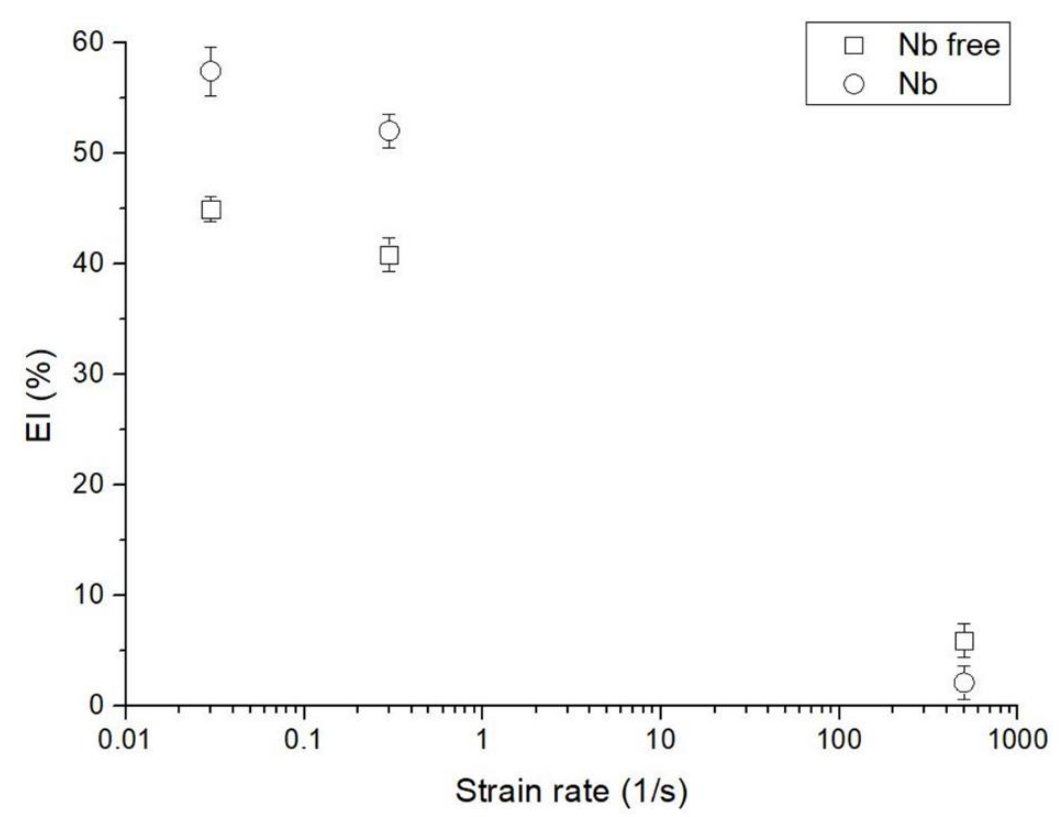

Figure 1: Embrittlement index for both the $\mathrm{Nb}$ and $\mathrm{Nb}$ free steel as function of the imposed strain rate.

\section{Conclusions}

The hydrogen susceptibility of Q\&P steels was investigated with emphasis on the effect of the addition of the microalloying element $\mathrm{Nb}$. The mechanical observations were explained 
via the use of the microstructural and hydrogen charging characterization. The main conclusions are the following:

- $\quad$ The more refined microstructure in micro-alloyed Q\&P steels leads to a higher hydrogen uptake capacity.

- The addition of $\mathrm{Nb}$ results in an increased hydrogen embrittlement in Q\&P steels. This is attributed to the higher hydrogen content as well as the higher fraction of low stability austenite.

- Limiting the diffusion time for hydrogen lowers the embrittlement effect. This explains the low susceptibility of Q\&P steels when tested at high strain rates.

- The suppression of the commonly beneficial TRIP effect, as a result of adiabatic heating, has a positive effect on the hydrogen embrittlement when tested under dynamic conditions.

The authors gratefully acknowledge the financial support received from the European Union's RFCS programme via the project OptiQPap, No. 709755.

\section{References}

[1] J. Speer, D. K. Matlock, B. C. De Cooman, and J. G. Schroth, "Carbon partitioning into austenite after martensite transformation," Acta Mater., vol. 51, no. 9, pp. 2611-2622, 2003.

[2] J. Mola and B. C. De Cooman, "Quenching and partitioning (Q\&P) processing of martensitic stainless steels," Metall. Mater. Trans. A Phys. Metall. Mater. Sci., vol. 44, no. 2, pp. 946-967, 2013.

[3] X. D. Wang, W. Z. Xu, Z. H. Guo, L. Wang, and Y. H. Rong, "Carbide characterization in a $\mathrm{Nb}$-microalloyed advanced ultrahigh strength steel after quenching-partitioning-tempering process," Mater. Sci. Eng. A, vol. 527, no. 15, pp. 3373-3378, 2010.

[4] F. Vercruysse, C. Celada-casero, B. M. Linke, P. Verleysen, and R. H. Petrov, "Temperature dependence of the static and dynamic behaviour in a Quenching and Partitioning processed low Si steel," Metals (Basel)., vol. 10, no. 4, p. 509, 2020.

[5] D. P. Koistinen and R. E. Marburger, "A general equation prescribing the extent of the austenite-martensite transformation in pure iron-carbon alloys and plain carbon steels," Acta Metall., vol. 7, pp. 59-60, 1959.

[6] C. Capdevila, F. G. Caballero, and C. García De Andrés, "Determination of Ms temperature in steels: A Bayesian neural network model," ISIJ Int., vol. 42, no. 8, pp. 894-902, 2002.

[7] M. A. Meyers, Dynamic Behavior of Materials. Hoboken, NJ, USA: John Wiley \& Sons, Inc, 1994.

[8] P. Verleysen, J. Degrieck, T. Verstraete, and J. Van Slycken, "Influence of Specimen Geometry on Split Hopkinson Tensile Bar Tests on Sheet Materials," Exp. Mech., vol. 48, no. 5, pp. 587-598, Oct. 2008.

[9] R. Zaera, J. A. Rodríguez-Martínez, and D. Rittel, “On the Taylor-Quinney coefficient in dynamically phase transforming materials. Application to 304 stainless steel," Int. J. Plast., vol. 40, pp. 185-201, 2013.

[10] F. Vercruysse, C. Celada-Casero, B. M. Linke, P. Verleysen, and R. H. Petrov, "The effect of $\mathrm{Nb}$ on the strain rate and temperature dependent behaviour of quenching \& partitioning steels," Mater. Sci. Eng. A, vol. 800, no. July 2020, p. 140293, 2021. 